\title{
Successful pregnancy in women with inferior vena cava stenosis - case report and discussion
}

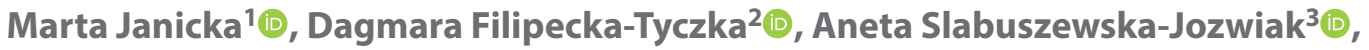 \\ Radoslaw Pietura ${ }^{4}$, , Grzegorz Jakiel ${ }^{3}$ (1) \\ 'Saint Sophia Hospital, Warsaw, Poland \\ ${ }^{2}$ Department of Reproductive Health, Centre of Postgraduate Medical Education, Warsaw, Poland \\ ${ }^{3} 1^{\text {st }}$ Department of Obstetrics and Gynecology, Centre of Postgraduate Medical Education, Warsaw, Poland \\ ${ }^{4}$ Department of Radiography, Lublin, Poland
}

\begin{abstract}
Objectives: Inferior vena cava syndrome (IVCS) is a heterogenous group of symptoms resulting in obstruction of the main vein inflow. Common reasons are thrombotic changes and tumors. Incidence of inferior vena cava (IVC) anomalies is $0.3 \%$ in general population. latrogenic IVC lesions caused by catheter insertion play increasing role. Treatment varies depending on the condition.

Material and methods: 32-year old patient was diagnosed with IVC stenosis during infertility related preconception evaluation and informed about increased risk in planned pregnancy. Throughout the well progressing pregnancy patient received low molecular weight heparin.

Results: The diagnosis was confirmed intraoperatively during the planned cesarean section. Early postpartum period was normal and patient was discharged with antithrombotic prophylaxis.

Conclusions: Isolated IVC stenosis in pregnancy has not been yet reported in medical literature. Even though IVC anomalies may be associated with other congenital changes, in this case the central venous line treatment in infancy seems to be the most likely cause. Malformations are often accidentally diagnosed because patients are usually asymptomatic. CT and MRI are recommended diagnostic tools. Conservative treatment is recommended for asymptomatic patients, as opposed to surgical treatment for symptomatic. However, due to condition's rarity, there is no evidence based approach management. Key words: inferior vena cava stenosis; inferior vena cava anomalies; varicose veins of parametrium; pregnancy complications

Ginekologia Polska 2020; 91, 6: 331-333
\end{abstract}

\section{INTRODUCTION}

The inferior vena cava (IVC) is the largest vein in the body with a diameter of 18-32 $\mathrm{mm}$. Inferior vena cava syndrome (IVCS) is a heterogeneous group of disorders causing obstruction of the inflow at various levels of the vein. It was well described by Sir William Osler as early as 1879 [1]. Development of IVC arises during the $4^{\text {th }}$ to $8^{\text {th }}$ week of gestation, and, due to its complexity, many malformations may appear [2]. Various IVC anomalies occur in approximately $0.3 \%$ of general population [3]. Sixty types of IVC malformations have been previously described [2], however, only few of them are clinically important. IVCS is often caused by thrombotic changes and tumors, and occurs rarely due to iatrogenic or genetic factors, aortic aneurysms, ascites, idiopathic retroperitoneal fibrosis, or retroperitoneal hematoma $[4,5]$.
Symptoms depend on the dynamics of the process and level of compression and may include edema of the lower extremities, varicose veins of the limbs, abdomen and chest, kidney failure, hepatomegaly, or pulmonary embolism. IVCS may appear in late pregnancy as a result of the enlarged gravid uterus compressing the IVC at supine position. This causes hypotension and may lead to stillbirth [6]. Treatment of IVCS varies depending on the primary condition. The authors present a rare case of an asymptomatic inferior vena cava stenosis and pelvic vein insufficiency during pregnancy which was found accidentally during infertility diagnosis.

\section{MATERIAL AND METHODS}

A 32-year-old woman presented at a tertiary Outpatient Clinic for preconception consultation due to infertility. As 
part of her medical history, the patient reported having been treated in infancy for sepsis in a neonatal intensive care unit with the use of a catheter placed in the IVC from the femoral access.

Congenital thrombophilia was excluded during infertility evaluation. Transvaginal ultrasound revealed extensive varicose veins of the pelvis and the patient was referred to a vascular surgeon. The angio-MR examination showed stenosis of vena cava inferior in the infrarenal section with subsequent significant widening of the pelvic veins, especially in the right parametrium (Fig. 1 and 2). Such anomaly could have been a congenital hypoplasia or a post-thrombotic lesion. However, in this case the suspected cause was the central venous line treatment in infancy. The right ovarian vein was enlarged with a cross-section diameter of $18 \times 16 \mathrm{~mm}$. Widening of the paraspinal veins in the lumbar region was also present. This condition is very rare and evidence-based approach management has not been yet established. Endovascular correction such as angioplasty with stent implantation prior to pregnancy was considered despite the increased risk of thrombosis perinatally and cardiogenic shock with arterial hypotension during labor. Ultimately surgical treatment was not deemed necessary. It was recommended that the patient visits a perinatology specialist immediately after becoming pregnant. The patient was also informed about the increased risk of pregnancy.

Before and during her pregnancy the patient did not exhibit any clinical signs of IVCS such as visible collateral circulation, edema or varicose veins of lower extremities. As the stenosis was asymptomatic, a conservative approach to treatment was selected. Initially the patient received antithrombotic prophylaxis with a preventive dose of $40 \mathrm{mg}$ of low molecular weight heparin (LMWH) and $75 \mathrm{mg}$ of acetylsalicylic acid. During the third trimester her activated partial thromboplastin time shortened and the LMWH dose was increased to $80 \mathrm{mg}$.

At 38 weeks of the pregnancy the patient was complaining of severe, intensifying at night, itching. Pregnancy cholestasis was suspected so she was admitted to the Saint Sofia Hospital. The physical examination revealed small scars on the skin of the upper and lower extremities where vessels catheterization was performed in childhood. While the pregnancy cholestasis diagnosis was confirmed, no symptoms or signs of IVCS were observed.

\section{RESULTS}

The patient was qualified for a cesarean section delivery due to ophthalmic reasons. A transverse, suprapubic, transperitoneal laparotomy was performed. The uterus was incised by the Cohen method. A healthy daughter of $3330 \mathrm{~g}$ and $55 \mathrm{~cm}$ was born, scoring 10 pts on the Apgar scale. Massive varicose veins of the parametrium were found intraoperatively. The vascular malformation of the vena cava

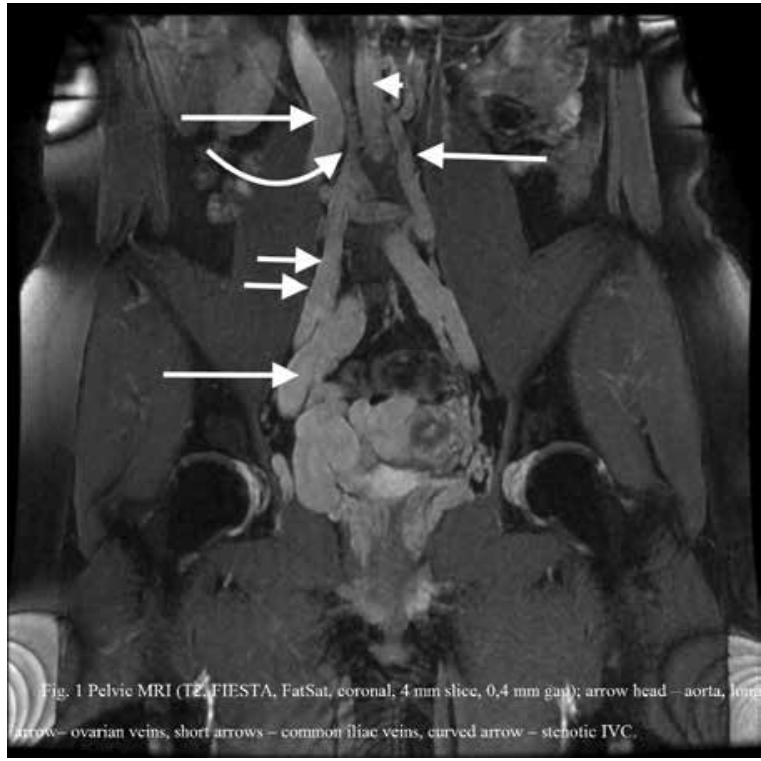

Figure 1. Pelvic MRI (T2, FIESTA, FatSat, coronal, $4 \mathrm{~mm}$ slice, $0.4 \mathrm{~mm}$ gap); arrowhead — aorta; long arrow — ovarian veins; short arrows — common iliac veins; curved arrow — stenotic IVC

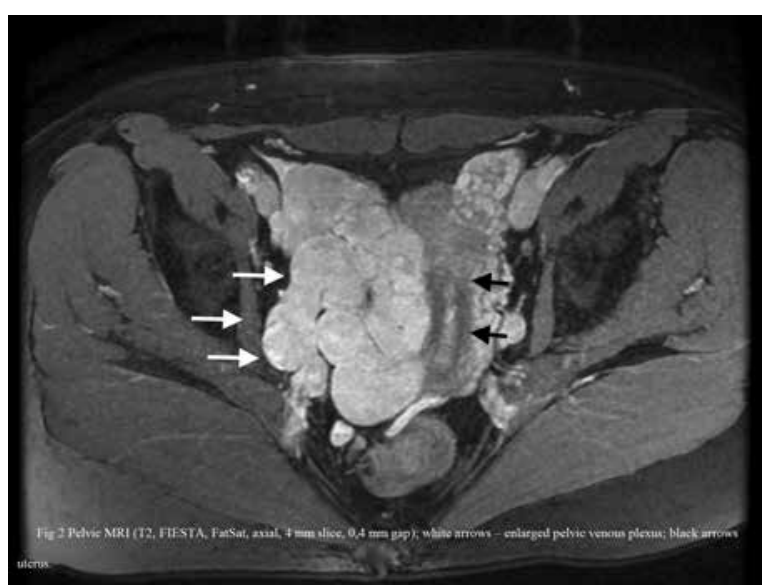

Figure 2. Pelvic MRI (T2, FIESTA, FatSat, axial, $4 \mathrm{~mm}$ slice, $0.4 \mathrm{~mm}$ gap); white arrows - enlarged pelvic venous plexus; black arrows uterus

was confirmed. The outside diameter of the malformed IVC was $10 \mathrm{~mm}$ width and its length being $5 \mathrm{~cm}$.

The early postpartum period was normal. Anticoagulant treatment with $80 \mathrm{mg}$ of LMWH was continued due to the thrombotic risk factors. The patient was discharged from the hospital on day 5 postpartum and antithrombotic prophylaxis was maintained for 6 weeks.

\section{DISCUSSION}

Isolated IVC stenosis in pregnancy has not been yet reported in medical literature. The main causes of IVCS are deep vein thrombosis and tumors. Absence of the infrarenal segment of the inferior vena cava is a rare anomaly. Since 1957 only 16 cases confirmed by imaging examinations have 
been reported in the medical literature [7-9]. Recent studies report that only $6 \%$ of IVC anomalies involved the renal or infrarenal segments [9]. Most of the congenital malformation are located suprarenal. Some authors suggest that the infrarenal absence of the inferior vena cava is embryonic in origin, others propose that it is the result of intrauterine or perinatal thrombosis $[9,10]$. In our opinion iatrogenic stenosis following a catheter insertion plays an increasing role. IVC anomalies are often diagnosed accidentally as patients are usually asymptomatic. Patients with other thromboembolism risk factors such as pregnancy, especially with caesarean section delivery may develop a lower limb thrombosis and pulmonary embolism. In developed countries this is the main cause of death during pregnancy $[9,11]$. The thrombotic risk is caused by pregnancy related hypercoagulability and stasis caused by the anomaly. The volume of the body fluids increases during pregnancy by up to $45 \%$. That is why the risk of cardiogenic shock with arterial hypotension during labor in this case was considered. IVC anomalies may also occur as pelvic congestion syndrome with chronic pain. In such cases IVC stenting followed by classic coil embolization to ovarian veins might be viable option as the gonadal veins may be the main source of collateral flow back to the central venous system [12]. Inferior vena cava anomalies may be associated with other congenital anomalies, especially of the spleen and liver [8, 9]. Computer tomography and magnetic resonance imaging are the recommended tests in the IVCS diagnosis $[9,13]$.

\section{CONCLUSIONS}

The management of IVC stenosis should depend on the condition and clinical symptoms. Conservative treatment is an option for asymptomatic patients. Thromboembolism risk can be managed with $\mathrm{LMWH}$ with the dose depending on all co-occurring rick factors. In symptomatic cases endovascular treatment is recommended [13]. A percutaneous balloon angioplasty with or without stent can be performed $[12,14,15]$. Evidence based approach management has not been yet developed as the condition is very rare [16]. Other thromboembolism risk factors should be excluded prior to conception and pregnancy in women with asymptomatic inferior vena cava stenosis should be carefully monitored. Antithrombotic prophylaxis should be implemented form the first trimester. Vaginal birth is recommended provided there are no other indications for cesarean section and immediate access to emergency treatment is ensured.

\section{Conflict of interest}

The Authors declare no conflict of interest.

\section{REFERENCES:}

1. Hartley JW, Awrich AE, Wong J, et al. Diagnosis and treatment of the inferior vena cava syndrome in advanced malignant disease. Am J Surg. 1986; 152(1): 70-74, doi: 10.1016/0002-9610(86)90145-5, indexed in Pubmed: 3728821.

2. Spentzouris G, Zandian A, Cesmebasi A, et al. The clinical anatomy of the inferior vena cava: a review of common congenital anomalies and considerations for clinicians. Clin Anat. 2014; 27(8): 1234-1243, doi: 10.1002/ca.22445, indexed in Pubmed: 25042045.

3. Hashmi ZA, Smaroff GG. Dual inferior vena cava: two inferior vena cava filters. Ann Thorac Surg. 2007; 84(2): 661-663, doi: 10.1016/j.athoracsur.2007.03.076, indexed in Pubmed: 17643661.

4. Sonin AH, Mazer MJ, Powers TA. Obstruction of the inferior vena cava: a multiple-modality demonstration of causes, manifestations, and collateral pathways. Radiographics. 1992; 12(2): 309-322, doi: 10.1148/radiographics.12.2.1561419, indexed in Pubmed: 1561419.

5. Vaglio A, Maritati F. Idiopathic Retroperitoneal Fibrosis. J Am Soc Nephrol. 2016; 27(7): 1880-1889, doi: 10.1681/ASN.2015101110, indexed in Pubmed: 26860343.

6. Humphries A, Stone P, Mirjalili SA. The collateral venous system in late pregnancy: A systematic review of the literature. Clin Anat. 2017; 30(8): 1087-1095, doi: 10.1002/ca.22959, indexed in Pubmed: 28726308.

7. Ramanathan T, Hughes TM, Richardson AJ. Perinatal inferior vena cava thrombosis and absence of the infrarenal inferior vena cava. J Vasc Surg. 2001; 33(5): 1097-1099, doi: 10.1067/mva.2001.114205, indexed in Pubmed: 11331855.

8. Bass JE, Redwine MD, Kramer LA, et al. Absence of the infrarenal inferior vena cava with preservation of the suprarenal segment as revealed by CT and MR venography. AJR Am J Roentgenol. 1999; 172(6): 1610-1612, doi: 10.2214/ajr.172.6.10350299, indexed in Pubmed: 10350299.

9. Salgado Ordóñez F, Gavilán Carrasco JC, Bermúdez Recio FJ, et al. Absence of the inferior vena cava causing repeated deep venous thrombosis in an adult--a case report. Angiology. 1998; 49(11): 951-956, doi: 10.1177/000331979804901113, indexed in Pubmed: 9822054.

10. Tofigh AM, Coscas R, Koskas F, et al. Surgical management of deep venous insufficiency caused by congenital absence of the infrarenal inferior vena cava. Vasc Endovascular Surg. 2008; 42(1): 58-61, doi: 10.1177/1538574407306791, indexed in Pubmed: 18238869.

11. Chee YL, Culligan DJ, Watson HG. Inferior vena cava malformation as a risk factor for deep venous thrombosis in the young. Br J Haematol. 2001; 114(4): 878-880, doi: 10.1046/j.1365-2141.2001.03025.x, indexed in Pubmed: 11564079.

12. Jurga-Karwacka A, Karwacki GM, Schoetzau A, et al. A forgotten disease: Pelvic congestion syndrome as a cause of chronic lower abdominal pain. PLoS One. 2019; 14(4): e0213834, doi: 10.1371/journal.pone.0213834, indexed in Pubmed: 30939134.

13. Collot J, Bletard N, Lamproye A. [Membranous occlusion of the inferior vena cava a rare cause of Budd-Chiari syndrome]. Rev Med Liege. 2018; 73(11): 557-561, indexed in Pubmed: 30431244.

14. Ding PX, Han XW, Liu C. Type II Abernethy Malformation in a Patient with Primary Budd-Chiari Syndrome. Ann Hepatol. 2019; 18(1): 246-249, doi: 10.5604/01.3001.0012.7933, indexed in Pubmed: 31113600.

15. Srinivas BC, Dattatreya PV, Srinivasa KH, et al. Inferior vena cava obstruction: long-term results of endovascular management. Indian Heart J. 2012; 64(2): 162-169, doi: 10.1016/S0019-4832(12)60054-6, indexed in Pubmed: 22572493.

16. Haddad RA, Saadaldin M, Kumar B, et al. Deep Vein Thrombosis Provoked by Inferior Vena Cava Agenesis. Case Rep Vasc Med. 2015; 2015: 651436, doi: 10.1155/2015/651436, indexed in Pubmed: 26788400. 